\title{
Interstellar grains: Effect of inclusions on extinction
}

\author{
Nisha Katyal ${ }^{1}$, Ranjan Gupta $^{1}$, and D. B. Vaidya ${ }^{2}$ \\ ${ }^{1}$ IUCAA, Post Bag 4, Ganeshkhind, Pune-411007, India \\ ${ }^{2}$ ICCSIR, Ahmedabad 380006, India
}

(Received August 31, 2010; Revised June 16, 2011; Accepted June 17, 2011; Online published February 2, 2012)

\begin{abstract}
A composite dust grain model which simultaneously explains the observed interstellar extinction, polarization, IR emission and the abundance constraints, is required. We present a composite grain model, which is made up of a host silicate oblate spheroid and graphite inclusions. The interstellar extinction curve is evaluated in the spectral region 3.4-0.1 $\mu \mathrm{m}$ using the extinction efficiencies of composite spheroidal grains for three axial ratios. Extinction curves are computed using the discrete dipole approximation (DDA). The model curves are subsequently compared with the average observed interstellar extinction curve and with an extinction curve derived from the IUE catalogue data.
\end{abstract}

Key words: Interstellar dust, extinction.

\section{Introduction}

It is highly unlikely that interstellar grains are spherical in shape or that they are homogeneous in composition and structure. Collected interplanetary particles are nonspherical and highly porous and composites of very small subgrains glued together (Brownlee, 1987). The existence of interstellar polarization requires that interstellar grains must be nonspherical. The elemental abundances derived from observed interstellar extinction also do not favour a homogeneous composition of interstellar grains. There is no exact theory to study light scattering by inhomogeneous grains (viz. porous, fluffy and composite). We have used Discrete Dipole Approximation (DDA) to study the extinction properties of the composite grains. For a description of DDA, see Draine (1988). In the present study, we calculate the extinction efficiencies for composite oblate spheroidal grains, made up of the host silicate spheroid with embedded inclusions of graphite, in the wavelength region 3.4-0.10 $\mu \mathrm{m}$. Using these extinction efficiencies of the composite grains with a power-law grain size distribution, we evaluate the interstellar extinction curve. We also estimate the cosmic abundances, viz. silicon and carbon, for the grain models which fit the observed interstellar extinction curve. It must be mentioned here that the composite oblate grain model presented in this study has also been used to interpret the observed IR emission from circumstellar dust (Vaidya and Gupta, 2011).

In Section 2, we give the validity criteria for the DDA and the composite oblate grain models. In Section 3, we present the results of our computations and discuss them. The main conclusions of our study are given in Section 4.

Copyright (c) The Society of Geomagnetism and Earth, Planetary and Space Sciences (SGEPSS); The Seismological Society of Japan; The Volcanological Society of Japan; The Geodetic Society of Japan; The Japanese Society for Planetary Sciences; TERRAPUB.

doi:10.5047/eps.2011.06.031

\subsection{Composite grains and DDA}

In the Discrete Dipole Approximation (DDA), a solid particle is replaced (approximated) by an array of $N$ dipoles. When a grain is exposed to an electromagnetic wave, each dipole responds to the radiation field of the incident wave as well as to the fields of the other $N-1$ dipoles that comprise the grain (Draine, 1988).

We use the computer code developed by Dobbie (see Vaidya et al., 2001) to generate the composite oblate grain models used in the present study. The constituent materials of the composite grains consist of silicates and graphites, since, in the interstellar medium, carbon and silicate occur separately and in the form of small particles which agglomerate into large grains. For a detailed discussion of the composition of the composite interstellar dust, see Mathis (1996) and Vaidya et al. (2001). We have studied composite grain models with a host silicate spheroid containing $N=9640,25896$ and 14440 dipoles, each carved out from $32 \times 24 \times 24,48 \times 32 \times 32$ and $48 \times 24 \times 24$ dipole sites, respectively; sites outside the spheroid are set to be vacuum and sites inside are assigned to be the host material. It is to be noted that the composite oblate spheroidal grain with $N=9640$ has an axial ratio $(A R)$ of 1.33 , whereas $N=25896$ has an axial ratio of 1.5 and $N=14440$ has an axial ratio of 2.0. Further, if the semi-major axis and the semi-minor axis are denoted by $x / 2$ and $y / 2$, respectively, then $a^{3}=(x / 2)(y / 2)^{2}$, where $a$ is the radius of the sphere whose volume is the same as the spheroid. To study randomly-oriented spheroids, it is necessary to get the scattering properties of the composite grains over all possible orientations. We use three values of each of the orientation parameters $(\beta, \theta$ and $\phi)$, i.e. averaging over 27 orientations, which we find is quite adequate (see Wolf $e t$ al., 1994). The volume fractions of the graphite inclusions used are $10 \%, 20 \%$ and $30 \%$ (denoted as $f=0.1,0.2$ and 0.3 ). The size of the inclusion is given by the number of dipoles, ' $n$ ' across the diameter of an inclusion; e.g. 152 

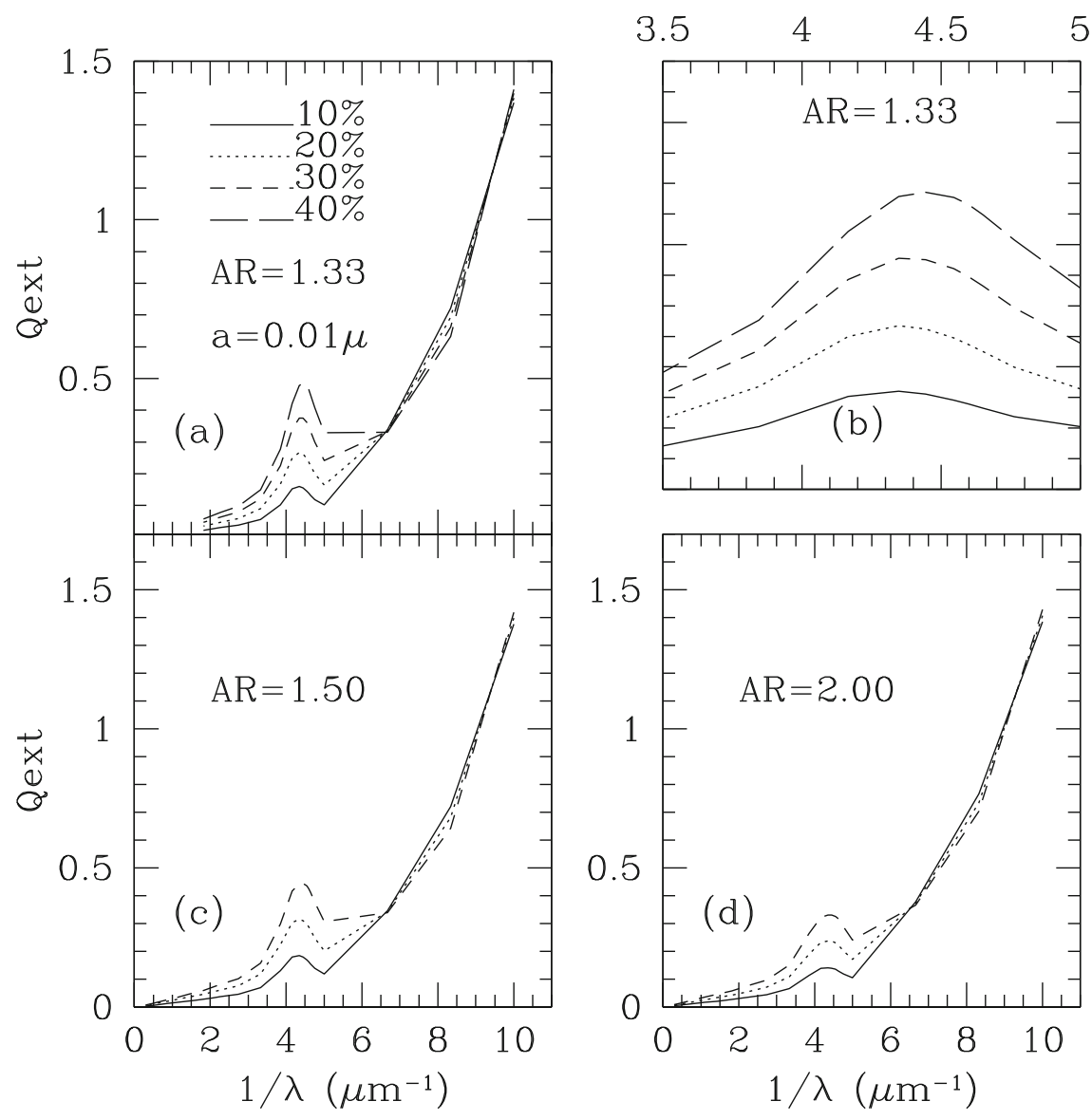

Fig. 1. Extinction efficiencies for the composite grains of size $0.01 \mu$ with host spheroids containing dipoles $N=9640,25896$ and 14440 ; corresponding to an axial ratio of 1.33, 1.50 and 2.00, respectively, are shown in (a), (c) and (d) in the wavelength region, 3.4-0.10 $\mu \mathrm{m}$ for three graphite inclusion fractions $(f=0.1,0.2$ and 0.3 corresponding to $10 \%, 20 \%$ and $30 \%$ inclusion fractions). An additional $40 \%$ inclusion case for $A R=1.33$ ( $N=9640$ ) is shown in the panels (a) and (b). In panel (b) the extinction curves in the wavelength region $0.28-0.20 \mu \mathrm{m}$ are highlighted.

for the composite grain model having $N=9640$, i.e. $A R$ $=1.33$ (see table I in Vaidya and Gupta, 2011). Details of the computer code and the corresponding modification to the DDSCAT 6.1 code (Draine and Flatau, 2003) are given in Vaidya et al. (2001) and Gupta et al. (2006). The modified code outputs a three-dimensional matrix specifying the material type at each dipole site; the sites are either silicate, graphite or vacuum. For an illustrative example of a composite oblate spheroidal grain with $N=14440$ dipoles $(A R=2.00)$, please refer to figure 1 of Gupta et al. (2006). There are two validity criteria for DDA (see e.g. Wolff et al., 1994); viz. (i) $|m| k d \leq 1$, where $m$ is the complex refractive index of the material, $k=2 \pi / \lambda$ is the wavenumber and $d$ is the lattice dispersion spacing, and (ii) $d$ should be small enough ( $N$ should be sufficiently large) to describe the shape of the particle satisfactorily. The complex refractive indices for silicates and graphite are obtained from Draine $(1985,1987)$. For each of the composite grain models, with $N=9640,25896$ and 14440 (i.e. $A R=1.33,1.50$ and 2.00 , respectively) and for all the grain sizes, between $a=0.001-0.250 \mu$, in the wavelength range of 3.4-0.1 $\mu \mathrm{m}$, considered in the present study; we have checked that the DDA criteria are satisfied (Vaidya et al., 2007).

\section{Results}

\subsection{Extinction efficiency of composite grains}

In the present paper, we study the extinction properties of spheroidal grains with three axial ratios $(A R)$, viz. 1.33, 1.5 and 2.0, corresponding to grain models with $N=9640$, 25896 and 14440 respectively, for three volume fractions of inclusions; viz. $10 \%, 20 \%$ and $30 \%$, in the wavelength region 3.4-0.10 $\mu \mathrm{m}$. Figures 1(a), (c) and (d) show the extinction efficiencies $\left(Q_{\text {ext }}\right)$ for the composite grains with the host silicate spheroids containing $N=9640,25896$ and 14440 dipoles, corresponding to an axial ratio 1.33 , 1.5 and 2.0, respectively, with a host composite grain size set to $a=0.01 \mu$. The three volume fractions, viz. $10 \%$, $20 \%$ and $30 \%$, of graphite inclusions are also listed in the top (a) panel and an additional volume fraction of $40 \%$ is also displayed. The extinction in the spectral region $0.28-0.20 \mu \mathrm{m}$ is highlighted in panel (b) of this figure for composite grains having $N=9640$.

The effect of the variation of the volume fraction of inclusions is clearly seen for all the models. The extinction efficiency increases as the volume fraction of the graphite inclusion increases. It is to be noted that the wavelength of the peak extinction shifts with the variation in the volume fraction of inclusions. These extinction curves also show the variation in the width of the extinction feature with the volume fraction of inclusions. All these results indicate 

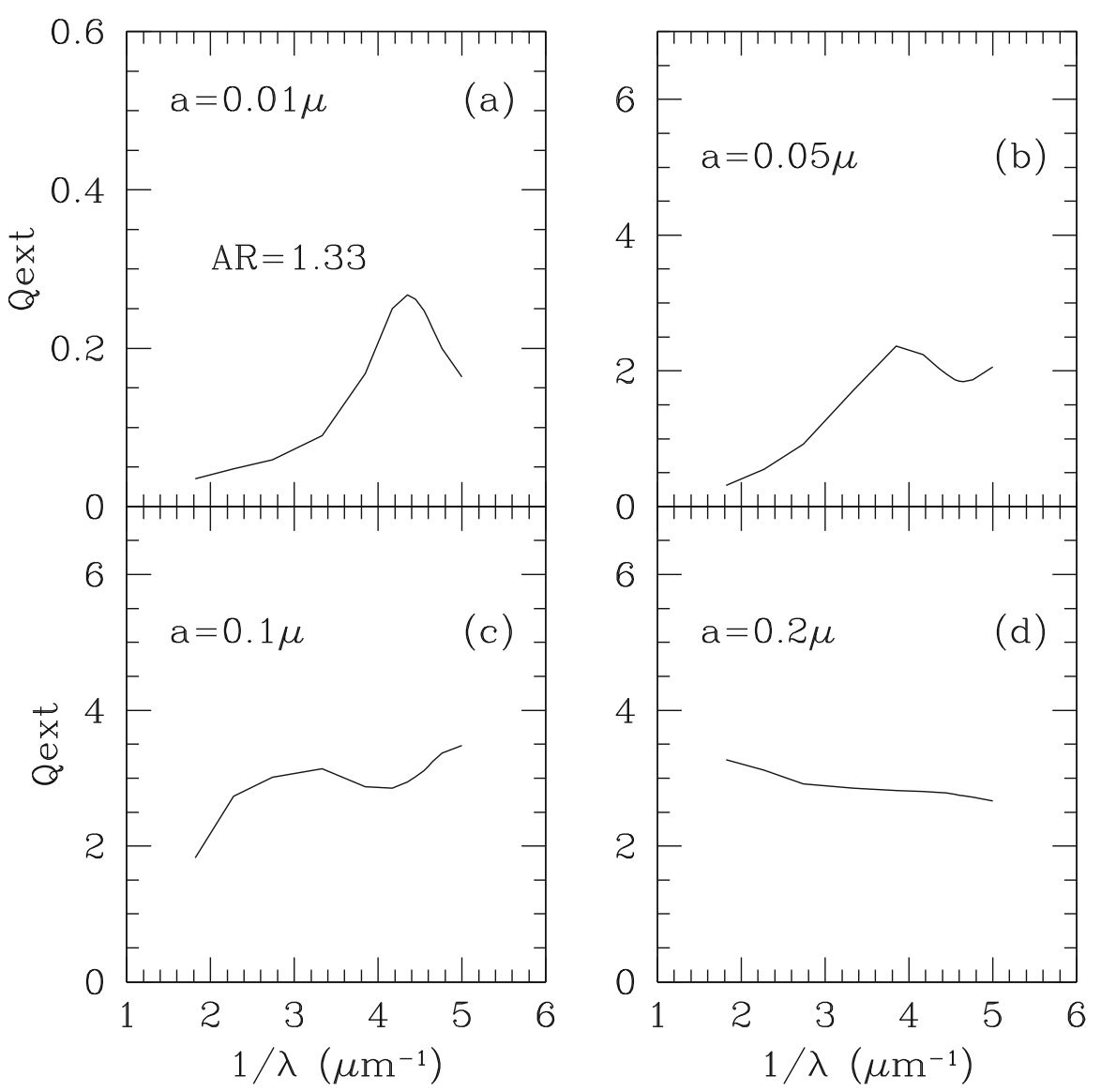

Fig. 2. Extinction efficiencies for the composite grains with $A R=1.33(N=9640)$ and with a $20 \%$ volume fraction of graphite inclusions for various grain sizes.

that the inhomogeneities within the grains play an important role in modifying the ' $2175 \AA$ ' feature. Voshchinnikov (1990) and Gupta et al. (2005) had found variations in the ' $2175 \AA$ ' feature with the shape of the grain, and Iati et al. (2001, 2004); Voshchinnikov (2002); Voshchinnikov and Farafanov (1993) and Vaidya et al. (1997, 1999) had found a variation in the feature with the porosity of the grains. Draine and Malhotra (1993) have found relatively little effect on either the central wavelength or the width of the feature for coagulated graphite silicate grains. Figures 2(ad) show the extinction efficiencies $\left(Q_{\text {ext }}\right)$ for the composite grains for four host grain sizes: viz. $a=0.01,0.05,0.1$ and $0.2 \mu$ at a constant volume fraction of inclusion of $20 \%$. It is seen that the extinction and the shape of the extinction curves varies considerably as the grain size increases. The ' $2175 \AA$ feature' is clearly seen for small grains; viz. $a=0.01$ and $0.05 \mu$, whereas for larger grains the feature almost disappears.

\subsection{Interstellar extinction curve}

The interstellar extinction curve (i.e. the variation of extinction with wavelength) is usually expressed by the ratio $E(\lambda-V) / E(B-V)$ versus $1 / \lambda$. We use the extinction efficiencies of the composite grains, with a power-law size distribution (i.e. $n(a) \sim a^{-3.5}$ ) (Mathis et al., 1977), to evaluate the interstellar extinction curve in the wavelength region of 3.4-0.10 $\mu \mathrm{m}$. In addition to the composite grains, a separate component of small graphite grains is required to produce the observed peak at $2175 \AA$ in the interstellar extinction curve (Mathis, 1996). The stability of the bump at $2175 \AA$ along all the lines of sight rules out the possibility of using just composite grains, made up of silicate with graphite as inclusions, to produce the bump (Iati et al., 2001).

The average observed interstellar extinction curve (Whittet, 2003) is then compared with the model curves formed from a $\chi^{2}$ minimized and best-fit linear combination of the composite and graphite grains (for details, see Vaidya and Gupta, 1999).

Figure 3(a) shows the interstellar extinction curve for the composite grains with $A R=1.33(N=9640)$ in the entire wavelength region of $3.4-0.10 \mu \mathrm{m}$ for the MRN grain-size distribution, with the size range, $a=0.005-0.250 \mu$. It is seen that composite spheroidal grain models with $A R=$ $1.33(N=9640)$ and $f=0.1$ fits the average observed extinction curve reasonably well in the wavelength range considered, i.e. $3.4-0.10 \mu \mathrm{m}$. The model extinction curves with $A R=1.50$ and $2.00(N=25896$ and 14440 , respectively) deviate from the observed extinction curve in the uv region, beyond the wavelength $\sim 0.1500 \mu \mathrm{m}$ (i.e. $6 \mu \mathrm{m}^{-1}$ ) and are thus not shown in the figure. These results with the composite grains indicate that spheroidal grains with an axial ratio not very large, i.e. $A R \sim 1.33(N=9640)$ is an optimum choice. The results indicate that a third component of very small grains (e.g. very small silicate grains or PAHs) may be required to explain the extinction beyond $1500 \AA$ in the UV (Weingartner and Draine, 2001). 

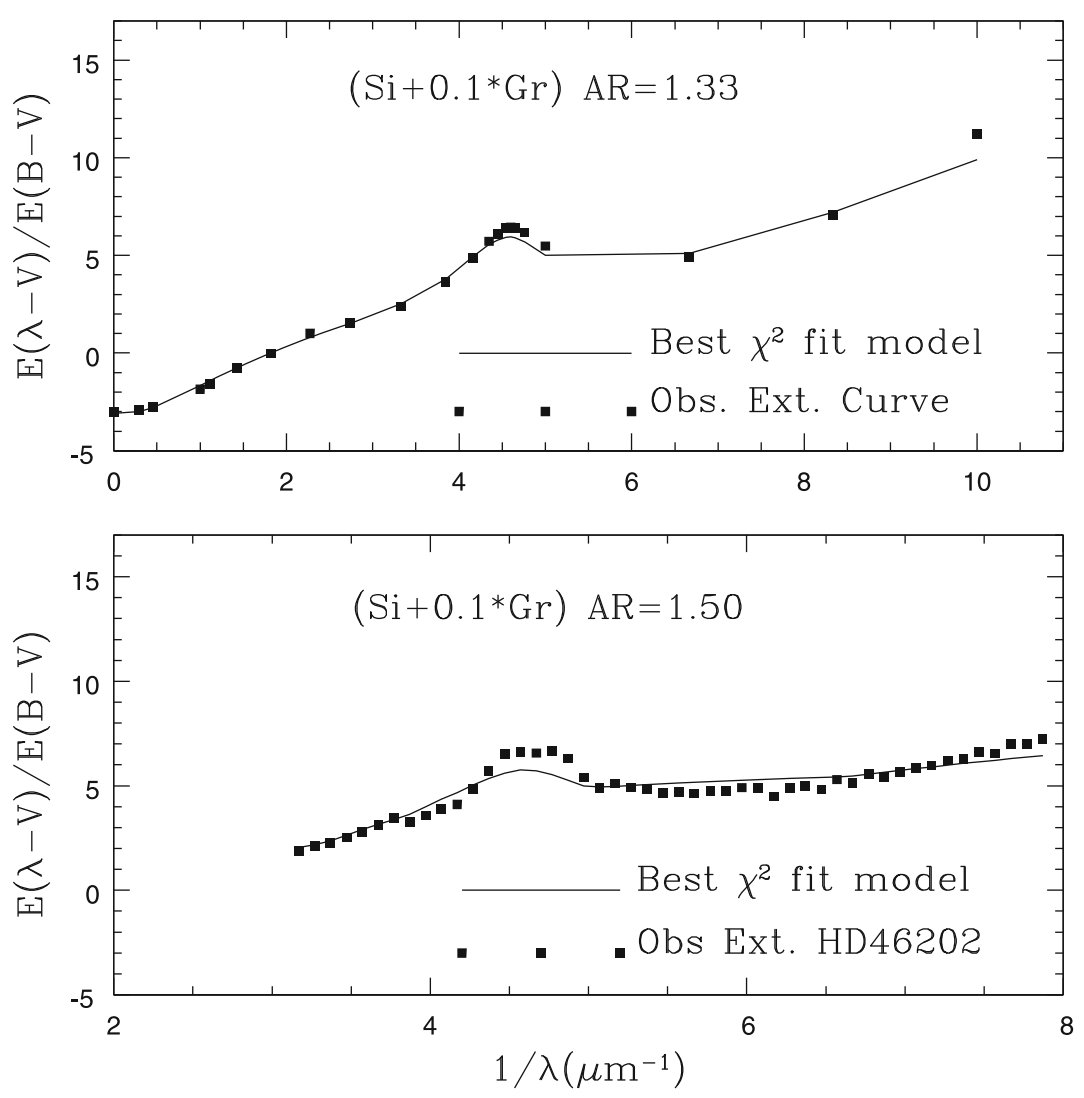

Fig. 3. (a) Comparison of the observed interstellar extinction curve (Whittet, 2003) with the best-fit model curve of composite grains with graphite inclusions in the wavelength range of 3.4-0.1 $\mu \mathrm{m}$. (b) Observed extinction curve in the direction of the star HD46202 and its comparison with the model curve of $A R=1.50$

In Fig. 3(b), we have displayed the observed extinction curve in the direction of the star HD46202 (data taken from IUE data base) and its best fitting with the model $A R=1.50$ $(N=25896)$ and grain-size distribution of $a=0.001-$ $0.100 \mu$. We have selected this particular star with $R_{v}=$ 3.1 , from our recent analysis of extinction curves towards the directions of 27 IUE stars (Katyal et al., 2011)

Recently, Iati et al. (2004), Zubko et al. (2004), Voshchinnikov et al. (2005) and Maron and Maron (2005) have also proposed composite grain models. Very recently, Voshchinnikov et al. (2006) have proposed composite porous grain models with three or more grain populations and have used both EMT-Mie type and layered sphere calculations.

\subsection{Cosmic abundances}

In addition to reproducing the interstellar extinction curve, any grain model must also be consistent with the abundance constraints. Snow and Witt $(1995,1996)$ have reviewed several models for the interstellar dust, which provide the data on the quantities of some elements that are required to reproduce the interstellar extinction. The number of atoms (in ppm) of the particular material tied up in grains can be estimated if the atomic mass of the element in the grain material and the density of the material are known (see, e.g., Cecchi-Pestellini et al. (1995) and Iati et al. (2001)). From the composite grain models we have proposed, we estimate $\mathrm{C}$ abundance i.e. $\mathrm{C} / \mathrm{H}$ between $\sim 165-$ 200 (including those atoms that produce the $2175 \AA$ feature), which is considerably lower than what is predicted by bare silicate/graphite grain models (e.g. C/H $254 \mathrm{ppm}$, $\mathrm{Li}$ and Draine, 2001) but it is still significantly above the ISM value of $\sim 110$ (Mathis, 2000); 140 (Sofia and Meyer, 2001) and 100 (Sofia and Parvathy, 2009). The estimated $\mathrm{Si}$ abundance from the composite grain model presented here is between 25 and 30, which is lower than the other grain models, 32 ppm (Li and Draine, 2001) and is consistent with the recent ISM value of $25 \mathrm{ppm}$ derived by Voshchinnikov and Henning (2010). For appropriate references on abundance standards and related topics, see Snow (2000) and Draine (2003).

\section{Summary and Conclusions}

Using the discrete dipole approximation (DDA) we have studied the extinction properties of composite spheroidal grains, made up of host silicate and graphite inclusions in the wavelength region of $3.4-0.10 \mu \mathrm{m}$. Our main conclusions from this study are:

(1) The extinction curves for the composite spheroidal grains show a shift in the central wavelength of the extinction peak as well as a variation in the width of the peak with a variation in the volume fraction of the graphite inclusions. These results clearly indicate that the shape, structure and inhomogeneity in the grains play an important role in producing the extinction. It must be noted here that large PAH molecules are also candidates for the carrier of the interstellar $2175 \AA$ feature - a natural extension of the graphite 
hypothesis (Draine, 2003).

(2) The extinction curves for composite spheroidal grains having an axial ratio not very large $(A R \sim 1.33, N=$ 9640 ) and $10 \%$ volume fractions of graphite inclusions are found to fit the average observed interstellar extinction satisfactorily. Extinction curves with other composite grain models with $N=25896$ and 14440 (i.e. with axial ratios of 1.50 and 2.00) deviate from the observed curves in the UV region, i.e. beyond about the wavelength $1500 \AA$. These results indicate that a third component of very small particles in the composite grains may help improve the fit in the UV region (see, e.g., Weingartner and Draine, 2001). It must be mentioned here that the composite spheroidal grain model with silicate and graphite as constituent materials proposed by us is not unique (see e.g. Zubko et $a l ., 2004)$. We have also attempted to fit models to the specific direction of the star HD46202 in our galaxy and show that $A R=1.50(N=25896)$ fits better in this case. Analysis is in progress for many more such directions in the galaxy.

(3) These results clearly show that the composite grain model is more efficient, compared to bare silicate/graphite grain models, in producing the extinction and it would perhaps help to reduce the cosmic abundance constraints. Composite grain models with silicate, graphite and an additional component (e.g. PAH's) may further reduce the abundance constraints.

We have used the composite spheroidal grain model to fit the observed interstellar extinction and have derived the abundance of carbon $(\mathrm{C} / \mathrm{H})$ and silicon $(\mathrm{Si} / \mathrm{H})$. The IRAS observations have indicated the importance of IR emission as a constraint on interstellar dust models (Zubko et al., 2004). Recently, we have used the composite spheroidal grain model to fit the IR emission curves obtained from IRAS observations (Vaidya and Gupta, 2011).

Acknowledgments. The authors thank the anonymous referee and N. V. Voshchinikkov for their useful comments in improving the manuscript. DBV thanks the organizing committee of the AOGS-2010, for providing the opportunity to present this paper at a meeting at Hyderabad, India, in July 2010. The authors acknowledge the financial support from the ISRO-Respond project (NO. ISRO/RES/2/2007-08).

\section{References}

Brownlee, D., in Interstellar Processes, edited by Hollenbach and Thompson H, Dordrecht and Reidel, 513, 1987.

Cecchi-Pestellini, C., S. Aiello, and B. Barsella, R V-dependent Interstellar Photodestruction Rates, Astrophys. J. Suppl., 100, 187, 1995.

Draine, B. T., Tabulated optical properties of graphite and silicate grains, Astrophys. J. Suppl., 57, 587, 1985.

Draine, B. T., Preprint Princeton Observatory, No. 213, 1987.

Draine, B. T., The discrete-dipole approximation and its application to interstellar graphite grains, Astrophys. J., 333, 848, 1988.

Draine, B. T., Interstellar dust grains, Ann. Rev. Astron. Astrophys., 41, 241, 2003.

Draine, B. T. and P. J. Flatau, User Guide for the Discrete Dipole Approximation Code DDSCAT.6.0, arXiv:astro-ph/0309069, 2003.

Draine, B. T. and S. Malhotra, On graphite and the $2175 \AA$ extinction profile, Astrophys. J., 414, 632, 1993.

Gupta, R., T. Mukai, D. B. Vaidya, A. Sen, and Y. Okada, Interstellar extinction by spheroidal dust grains, Astron. Astrophys., 441, 555, 2005.
Gupta, R., D. B. Vaidya, J. S. Dobbie, and P. Chylek, Scattering properties and composition of cometary dust, Astrophys. Space Sci., 301, 21, 2006.

Iati, M. A., C. Cecchi-Pestellini, D. A. Williams, F. Borghese, and P. Denti, Borghese F, Denti, P., Porous interstellar grains, Month. Not. R. Astron. Soc., 322, 749, 2001.

Iati, M. A., A. Giusto, R. Saija, F. Borghese, P. Denti, C. Cecchi-Pestellini, and S. Aielo, Optical properties of composite interstellar grains: A morphological analysis, Astrophys. J., 615, 286, 2004.

Katyal, N., R. Gupta, and D. B. Vaidya, Interstellar dust models towards some IUE stars, 2011 (in preparation).

Li, A. and B. T. Draine, Infrared emission from interstellar dust. II. The diffuse interstellar medium, Astrophys. J., 554, 778, 2001.

Maron, N. and O. Maron, On the mixing rules for astrophysical inhomogeneous grains, Month. Not. R. Astron. Soc., 357, 873, 2005.

Mathis, J. S., Dust models with tight abundance constraints, Astrophys. J., 472, 643, 1996.

Mathis, J. S., Properties of interstellar dust, J. Geophys. Res., 105, 10269 , 2000.

Mathis, J. S., W. Rumpl, and K. H. Nordsieck, The size distribution of interstellar grains, Astrophys. J., 217, 425, 1977.

Snow, T. P., Composition of interstellar gas and dust, J. Geophys. Res., 105, 10239, 2000.

Snow, T. P. and A. N. Witt, The interstellar carbon budget and the role of carbon in dust and large molecules, Science, 270, 1455, 1995.

Snow, T. P. and A. N. Witt, Interstellar depletions updated: Where all the atoms went, Astrophys. J., 468, L65, 1996.

Sofia, U. J. and D. M. Meyer, Interstellar abundance standards revisited, Astrophys. J., 554, L221, 2001.

Sofia, U. J. and V. S. Parvathy, Cosmic dust near and far, in ASP Conference Series, Vol. 414, 236, edited by Henning, Th., E. Grun, and J. Steinacker, 2009.

Vaidya, D. B. and R. Gupta, Extinction by porous silicate and graphite grains, Astron. Astrophys., 328, 634, 1997.

Vaidya, D. B. and R. Gupta, Interstellar extinction by porous grains, Astron. Astrophys., 348, 594, 1999.

Vaidya, D. B. and R. Gupta, Composite grains: Effects of porosity and inclusions on the silicate feature, J. Quant. Spectrosc. Rad. Transfer, 110, 1726, 2009.

Vaidya, D. B. and R. Gupta, Infrared emission from the composite grains: effects of inclusions and porosities on the 10 and $18 \mu \mathrm{m}$ features, Astron. Astrophys., 528, A57, 2011.

Vaidya, D. B., R. Gupta, J. S. Dobbie, and P. Chylek, Interstellar extinction by composite grains, Astron. Astrophys., 375, 584, 2001.

Vaidya, D. B., R. Gupta, and T. P. Snow, Composite interstellar grains, Month. Not. R. Astron. Soc., 379, 791, 2007.

Voshchinnikov, N. V., Interstellar Lambda 2200 Band and Aspherical Graphite Grains, Sov. Aston. Lett., 16(3), 215, 1990.

Voshchinnikov, N. V., in Optics of Cosmic Dust, edited by Videen, G. and M. Kocifaj, Kluwer, 3, 2002.

Voshchinnikov, N. V. and V. G. Farafanov, Optical properties of spheroidal particles, Astrophys. Space Sci., 204, 19, 1993.

Voshchinnikov, N. V. and Th. Henning, From interstellar abundances to grain composition: the major dust constituents $\mathrm{Mg}, \mathrm{Si}$, and $\mathrm{Fe}, 2010$, Astron. Astrophys., 517, A45, 2010.

Voshchinnikov, N. V., V. B. Il'in, and Th. Henning, Modelling the optical properties of composite and porous interstellar grains, Astron. Astrophys., 429, 371, 2005.

Voshchinnikov, N. V., V. B. Il'in, Th. Henning, and D. N. Dobkova, Dust extinction and absorption: the challenge of porous grains, Astron. Astrophys., 445, 993, 2006.

Weingartner, J. C. and B. T. Draine, Dust grain-size distributions and extinction in the Milky Way, large Magellanic cloud, and small Magellanic cloud, Astrophys. J., 548, 296, 2001.

Whittet, D. C. B., Dust in the Galactic Environments, 2nd edition, p. 76, IoP Publishing, Bristol, 2003.

Wolff, M. J., G. C. Clayton, P. G. Martin, and R. E. Sculte-Ladlback, Modeling composite and fluffy grains: The effects of porosity, Astrophys. J., 423, 412, 1994.

Zubko, V., E. Dwek, and R. G. Arendt, Interstellar dust models consistent with extinction, emission, and abundance constraints, Astrophys. $J$. Suppl., 152, 211, 2004.

N. Katyal (e-mail: nishakat@iucaa.ernet.in), R. Gupta, and D. B. Vaidya 